\title{
A wideband cryogenic microwave low-noise amplifier
}

\author{
Boris I. Ivanov ${ }^{*}$, Dmitri I. Volkhin¹, Ilya L. Novikov¹, Dmitri K. Pitsun ${ }^{1}$, \\ Dmitri O. Moskalev2,3, Ilya A. Rodionov ${ }^{2,3}$, Evgeni I''ichev ${ }^{1,4}$ and Aleksey G. Vostretsov ${ }^{1}$
}

Open Access

\author{
Full Research Paper \\ Address: \\ ${ }^{1}$ Novosibirsk State Technical University, K.Marx-Av.20, Novosibirsk, \\ 630073, Russia, ${ }^{2}$ FMN Laboratory, Bauman Moscow State Technical \\ University, 2-nd Baumanskaya str. 5, Moscow, 105005, Russia, \\ ${ }^{3}$ Dukhov Automatics Research Institute, (VNIIA), 22 ul. \\ Sushchevskaya, Moscow, Russia, 127055 and ${ }^{4}$ Leibniz Institute of \\ Photonic Technology, PO Box 100239, D-07702 Jena, Germany \\ Email: \\ Boris I. Ivanov* - to_ivanov_boris@yahoo.com \\ * Corresponding author

\section{Keywords:} \\ cryogenic low-noise amplifier; high-electron-mobility transistor \\ (HEMT); HEMT amplifier; microwave cryogenic amplifier; microwave \\ superconducting circuit readout; superconducting qubit readout
}

\begin{abstract}
Beilstein J. Nanotechnol. 2020, 11, 1484-1491. https://doi.org/10.3762/bjnano.11.131
\end{abstract}

Received: 30 May 2020

Accepted: 31 August 2020

Published: 30 September 2020

This article is part of the thematic issue "Functional nanostructures for electronics, spintronics and sensors".

Guest Editor: A. S. Sidorenko

(C) 2020 Ivanov et al.; licensee Beilstein-Institut. License and terms: see end of document.

\begin{abstract}
A broadband low-noise four-stage high-electron-mobility transistor amplifier was designed and characterized in a cryogen-free dilution refrigerator at the $3.8 \mathrm{~K}$ temperature stage. The obtained power dissipation of the amplifier is below $20 \mathrm{~mW}$. In the frequency range from 6 to $12 \mathrm{GHz}$ its gain exceeds $30 \mathrm{~dB}$. The equivalent noise temperature of the amplifier is below $6 \mathrm{~K}$ for the presented frequency range. The amplifier is applicable for any type of cryogenic microwave measurements. As an example we demonstrate here the characterization of the superconducting X-mon qubit coupled to an on-chip coplanar waveguide resonator.
\end{abstract}

\section{Introduction}

Quantum microwave devices are widely used for different applications ranging from radio astronomy [1-3] to quantum information processing circuits [4]. The latter include the most challenging and attractive topics such as quantum bits (qubits) [5], quantum dots [6], microwave single-photon detectors [7], highquality resonators [8], superconducting microwave beam splitters [9], and other circuit quantum electrodynamics structures aimed to be used as elements for quantum processors. The ex- perimental study of the abovementioned quantum devices requires precise low-noise readout electronics including lownoise amplifiers [10].

Two main low-noise amplifiers parameters are small equivalent noise temperature $\left(T_{\mathrm{n}}\right)$ and relatively high gain $(G)$. The superconducting qubit measurement setup requires particular lownoise amplifiers applicable for cryogenic temperatures. An im- 
portant point is that cryogenic low-noise amplifiers (cLNAs) are wideband noise sources itself. In the superconducting qubit experimental setup the noise generated from a cLNA is introduced to the sample, which might be crucial for the main qubit parameters, especially for the relaxation time $T_{1}$ and coherence time $T_{2}$. Therefore, cryogenic microwave isolators are used between the sample and the cLNA.

Most of the modern cryogenic amplifiers operate at temperatures of liquid helium [11-16] and are, usually, placed at the $4 \mathrm{~K}$ stage of dilution refrigerators. These amplifiers can be implemented using two modern semiconductor transistor technologies, that is, high-electron-mobility transistor (HEMT) technology, including GaAs and InP, and SiGe heterojunction bipolar technology (HBT). Recently, it has been shown that cryogenic amplifiers based on SiGe HBT [16-18] can provide low noise levels and high gain at $4 \mathrm{~K}$. They are suitable for superconducting qubit readout $[25,26]$ and radio astronomy systems $[12,14]$.

In order to obtain high gain values for $C$ (ranging from 4 to $8 \mathrm{GHz}$ ) and $X$ (ranging from 8 to $12 \mathrm{GHz}$ ) frequency bands the number of amplification stages of the HBT cLNA should be increased. This leads to an increase of the total power dissipation in the dilution refrigerator, which makes its operation unstable. In contrast, using modern HEMT technology allows one to implement cryogenic amplifiers with high gain values for a wide frequency range up to tens of gigahertz $[11,13,19]$. Most of them are based on monolithic microwave integrated circuit (MMIC) technology, which yields outstanding noise parameters in the $C$ and $X$ frequency bands. However, for some applications a flexible amplifier design is needed. Indeed, there are known schematic realizations of cLNAs based on discrete components $[11,17,20-24]$. The experimental characteristics of the presented amplifiers show that they do not provide gain values of more than $30 \mathrm{~dB}$ in the frequency range from 8 to $12 \mathrm{GHz}$. The evolving semiconductor technology provides the modern market with state-of-art commercially available transistors to substitute old types. A new type of commercially available transistors was used for the cLNA presented in this paper. A realization of a microwave frequency cLNA design, based on commercially available HEMT transistors, requires accurate and precise matching and an appropriate selection of passive components for liquid-helium temperatures.

In this paper we show that an accurate microwave matching circuit design based on commercially available transistors yields low-cost stable cryogenic low-noise amplifiers with a frequency range up to $12 \mathrm{GHz}$. The implemented cLNA has the following parameters: a gain value of more than $30 \mathrm{~dB}$ for a frequency range from 6 to $12 \mathrm{GHz}$ and an equivalent noise temper- ature below $6 \mathrm{~K}$. The cLNA has $50 \Omega$ input and output terminations and can be installed in a microwave measurement setup at the $4 \mathrm{~K}$ stage of modern dilution refrigerators. The implemented amplifier was tested by superconducting X-mon qubit measurements. In order to measure the $\mathrm{X}$-mon qubit parameters the standard low signal power one microwave tone $[25,26]$ and two microwave tone [27] spectroscopy experiments were performed.

\section{Results and Discussion Cryogenic four-stage low-noise amplifier design}

The amplifier design was optimized for low noise, adequate gain, and appropriate output matching at cryogenic temperatures. The latter is important for better matching with roomtemperature amplifiers. CE3512K2 GaAs pseudomorphic HEMT transistors made by CEL were used. The transistors were selected with regard to the two following parameters: minimum noise figure (NF) of $0.3 \mathrm{~dB}$ and minimum required value of associated gain of $12 \mathrm{~dB}$ at a frequency of $12 \mathrm{GHz}$. The design was optimized for the frequency range from 6 to $12 \mathrm{GHz}$, which fits to most of modern qubit measurement setups. We used the available S-parameters of the transistor for the matching circuit design. From a circuit simulation with ideal components a minimal gain value of more than $36 \mathrm{~dB}$ at $300 \mathrm{~K}$ in the frequency range from 6 to $12 \mathrm{GHz}$ was obtained. For the next step we used the real S-parameters of passive circuit components placed on a dielectric substrate. High-frequency edged trimmed block resistors with a 0402 package size from Vishay company were used in drain and gate biasing lines. The appropriate selection of the resistors in cryogenics is a crucial point in amplifier implementation. We should mention that the first stage of the amplifier does not have a gate resistor in its circuit. One of the reasons is a noise reduction, which is introduced to the input circuit of the amplifier. For increasing the circuit stability around $10 \mathrm{GHz}$ and better matching, $10 \Omega$ gate resistors were used for the second and the fourth stage. Multilayer ceramic capacitors with $\mathrm{C} 0 \mathrm{G}$ dielectric material in a 0402 package were used. Murata ceramic core coils with 0201 SMD package size and a self-resonance frequency of about $20 \mathrm{GHz}$ were used for gate and drain power supply circuits. All passive components, for example, microwave capacitors, coils, and precision resistors were measured before at liquid helium temperature for obtaining the spread of nominal parameters. The amplifier was assembled on a Rogers RO 4350 substrate and the matching circuits were carried out as microstrip lines.

The amplifier was mounted inside a brass box separated into two chambers. Each chamber contains two stages of the amplifier. The separation was made in order to avoid standing waves 


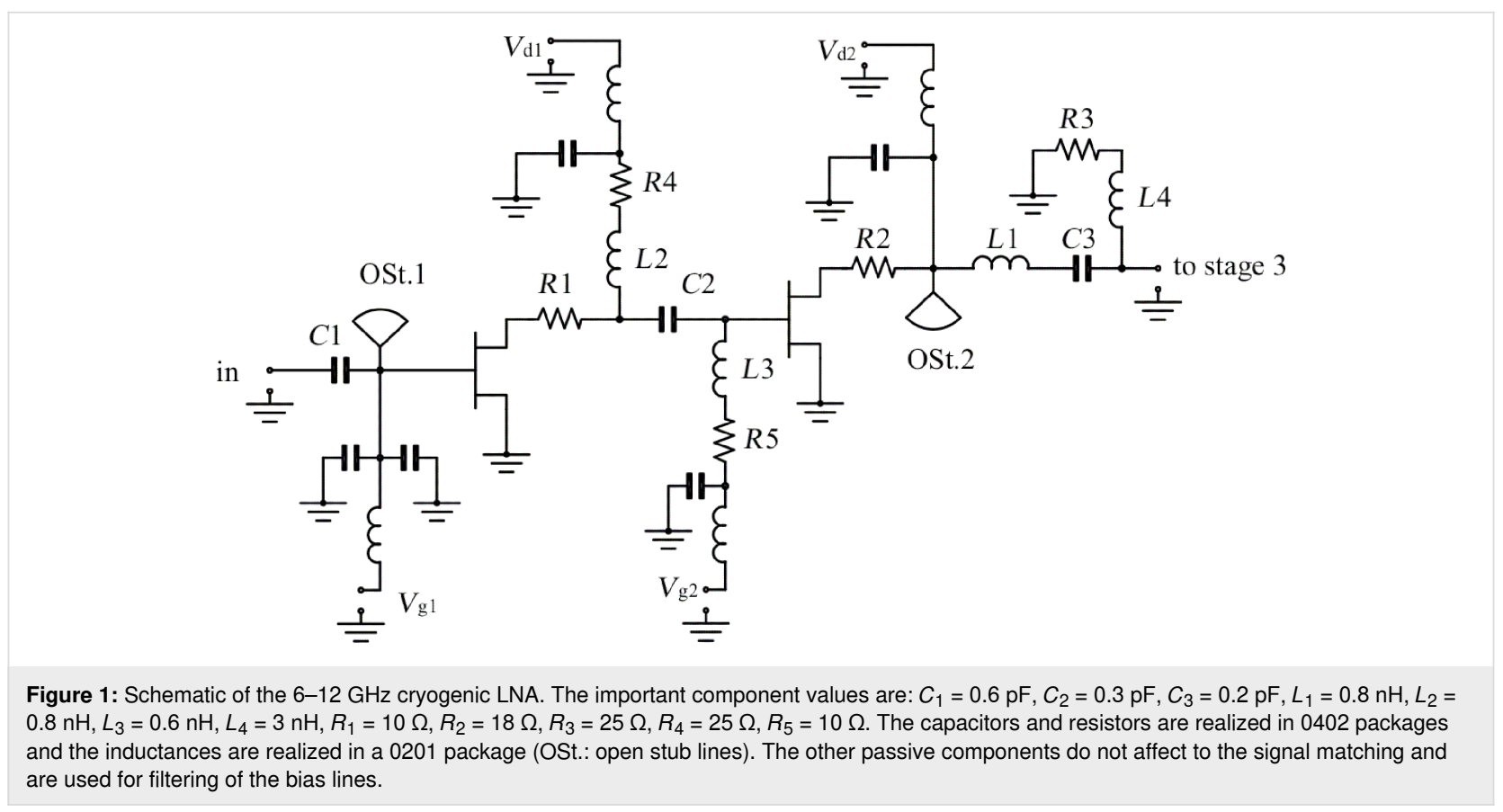

and self-resonances in the box in the operation frequency range. We did an electromagnetic simulation of the box and it showed us the absence of self-resonances in a frequency range from 1 to $12 \mathrm{GHz}$. The substrate was soldered directly to the brass box and SubMiniature version A (SMA) connectors with a specified frequency range up to $12 \mathrm{GHz}$ were soldered directly to the microwave laminate. The feedthrough filters were used as the input biasing electrodes and the power supply cables from a micro-D cryostat connector were soldered directly to them. The first two stages of the amplifier circuit are presented in Figure 1. The third and the fourth stage are identical. One of the key elements in the matching circuits is an open stub line (marked as OSt. in Figure 1) in the gate circuit of the input stage and in the drain circuit of the second and the fourth stage (Figure 2).

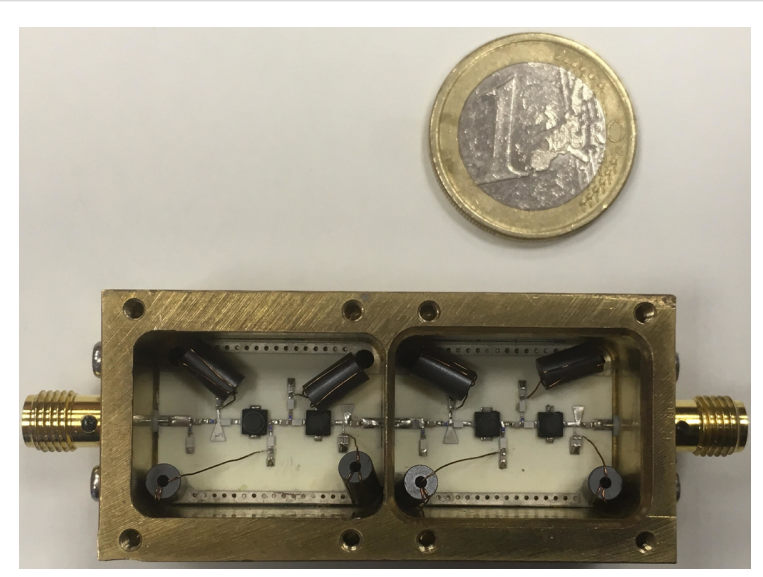

Figure 2: Realization of the cryogenic LNA.

\section{Cryogenic LNA characterization}

The measurement system of the amplifier was based on a $10 \mathrm{mK}$ closed-cycle dilution refrigerator where the amplifier was installed and fixed with screws to the copper holder at the $4 \mathrm{~K}$ plate. We used standard $2.2 \mathrm{~mm} \mathrm{CuBe} 50 \Omega$ coaxial microwave lines between $4 \mathrm{~K}$ stage and room-temperature SMA flange as input and output signal lines installed into the dilution refrigerator for the S-parameter and noise temperature measurements of the amplifier. The simplified measurement scheme is presented in Figure 3. In order to reduce the input signal level, to perform thermal anchoring at each stage, and to prepare the noise measurement setup, cryogenic broadband $10 \mathrm{~dB}$ and $20 \mathrm{~dB}$ attenuators were installed at the $50 \mathrm{~K}$ flange and the $4 \mathrm{~K}$ flange, respectively. Moreover, with the described setup we were able to perform gain and noise measurements during one cooldown without rebuilding the measurement scheme and warming up the cryostat. An additional channel with identical microwave coaxial cables and cryogenic attenuators was used for compensating the losses and calibrating the noise temperature. It is marked as satellite line in Figure 3. A short $2.2 \mathrm{~mm}$ $50 \Omega$ coaxial cable with SMA termination was used to connect the satellite lines. Gain and noise properties of the amplifier were characterized at the exact temperature of $3.8 \mathrm{~K}$.

We designed and implemented an independent eight-channel power supply unit based on $\mathrm{Pb}$ batteries, which allowed us to bias drain and gate circuits of each stage independently. The S-parameters of the designed cLNA and the satellite lines were measured using a vector network analyzer (VNA) in a frequency range from 4 to $13 \mathrm{GHz}$ and with an output signal power 


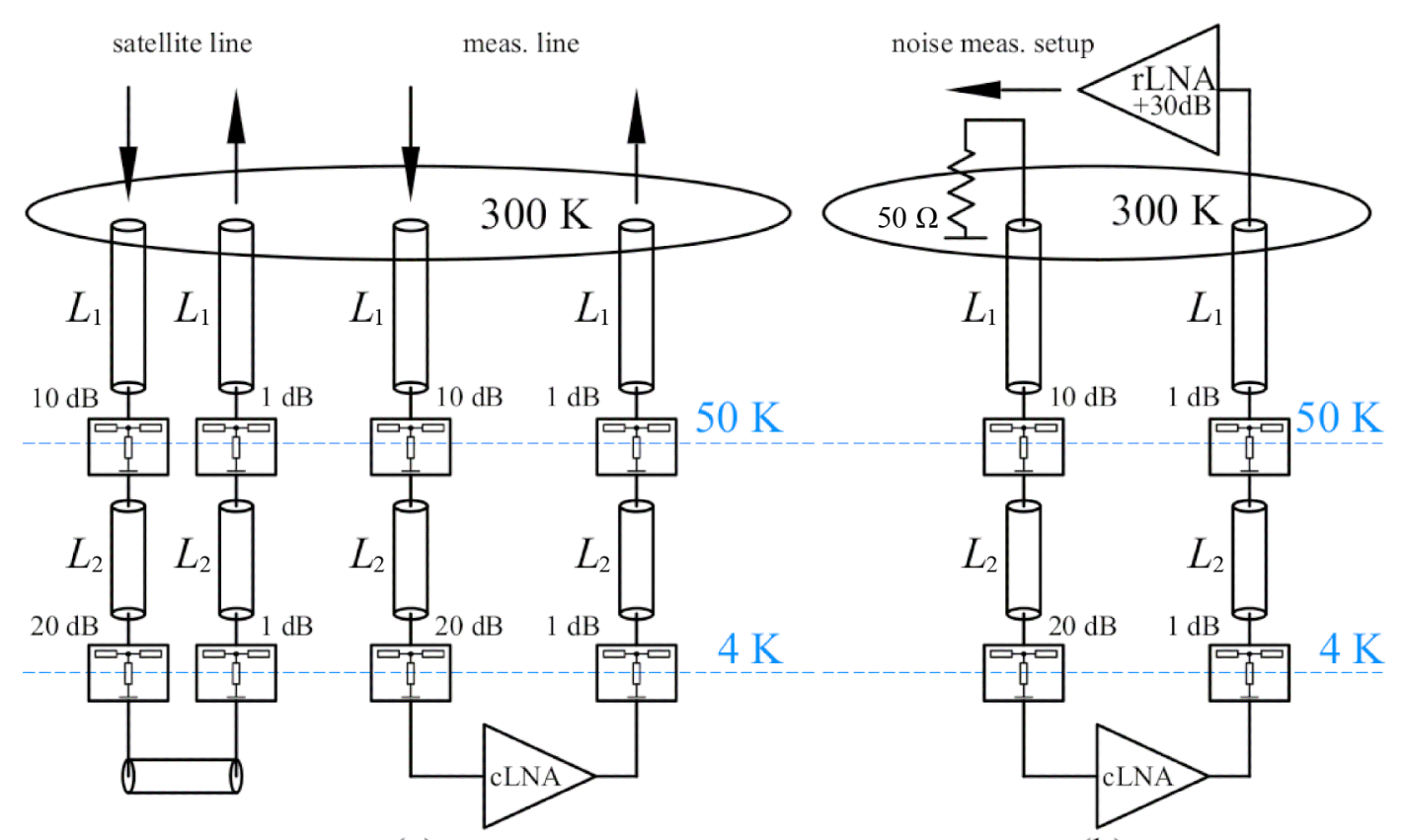

(a)

(b)

Figure 3: Noise and gain measurement setup. (a) Calibration lines (left-hand side sketch) and gain measurement setup (right-hand side sketch). (b) Noise measurement setup for the cryogenic LNA.

level of $-20 \mathrm{dBm}$. The total power applied to the input of the cLNA was lower than $-50 \mathrm{dBm}$. We performed transmission measurements of the satellite lines and calibrated our measurement setup according to the loss rate. The gain curve is presented in Figure 4. We were not able to measure the reflection coefficients during the cooldown because of the setup features. The output reflection of the cLNA $\left|S_{22}\right|$ was measured separately and the reflection level did not exceed $-10 \mathrm{dBm}$ for the presented frequency range.

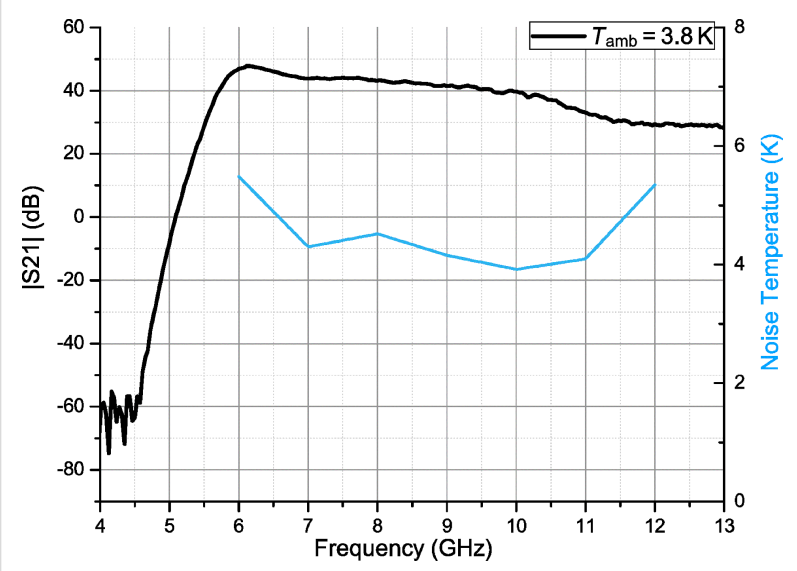

Figure 4: Gain and noise temperature of the cryogenic LNA at the experimental temperature of $3.8 \mathrm{~K}$.
The noise measurement procedure required an approach with higher precision. In this work we estimated the highest equivalent noise temperature level. We used the VNA in the spectrum analyzer mode and measured the power noise density at the output of the setup. In order to increase the sensitivity we applied an additional low-noise broadband room-temperature microwave amplifier (see rLNA in Figure 3a) directly to the $300 \mathrm{~K}$ cryostat SMA output flange. The gain $G_{2}$ and noise $P_{2}$ properties of the rLNA were characterized before by means of a calibrated noise source and the Y-factor procedure [28]. In order to terminate the input line we placed a broadband $50 \Omega$ load at the cryostat microwave input at the $300 \mathrm{~K}$ flange SMA connector, see Figure $3 \mathrm{~b}$. Using the setup shown in Figure $3 \mathrm{~b}$ we measured the total noise power density $P_{\text {total }}$ in the frequency range from 6 to $12 \mathrm{GHz}$. It is defined as

$$
P_{\text {total }}=P_{1} t+P_{2} G_{2}+P_{\mathrm{VNA}}
$$

where $P_{1}$ is the cLNA input power noise density, $P_{2} G_{2}$ is the output power noise density of the room-temperature low-noise amplifier, $P_{\mathrm{VNA}}$ is the spectrum analyzer self-noise, and $t$ is the transmission coefficient from the input of the cLNA to the output of the rLNA. It is defined as

$$
t=\frac{G_{1} G_{2}}{L_{\text {out }}},
$$


where $L_{\text {out }}$ is the loss rate of the output line calibrated previously. All variables are in the linear scale. The output line loss coefficient depends linearly on the frequency. First, we obtained the power noise density of the cryogenic LNA with an exception of rLNA noise and VNA noise, $P_{1} t=P_{\text {total }}-P_{2} G_{2}-$ $P_{\mathrm{VNA}}$. Then the power noise density related to the input, $P_{1}$, was obtained and the equivalent noise temperature was calculated according to the formula

$$
T_{\mathrm{Na}}=\frac{P_{1}}{k_{\mathrm{B}}} .
$$

The noise curve is presented in Figure 4 and the equivalent noise temperature does not exceed $6 \mathrm{~K}$ for the frequency range from 6 to $12 \mathrm{GHz}$. The minimum noise temperature of $4 \mathrm{~K}$ was obtained at $10 \mathrm{GHz}$. This can be explained by better noise matching at $4 \mathrm{~K}$ where the parameters of the passive components are slightly different. The amplifier parameters measured at $3.8 \mathrm{~K}$ are summarized in Table 1 . The amplifier exhibits low equivalent noise temperature and high gain. Therefore, it is suitable for the superconducting qubit readout.

Table 1: The main parameters of the cryogenic LNA.
\begin{tabular}{ccccc}
$\begin{array}{c}G_{\min } \\
{[\mathrm{dB}]}\end{array}$ & $\begin{array}{c}\text { BW } \\
{[\mathrm{GHz}]}\end{array}$ & $\begin{array}{c}\max T_{\text {noise }} \\
{[\mathrm{K}]}\end{array}$ & $\begin{array}{c}P_{\text {cons }} \\
{[\mathrm{mW}]}\end{array}$ & $\begin{array}{c}T_{\text {amb }} \\
{[\mathrm{K}]}\end{array}$ \\
\hline 30 & $6-12$ & 6 & 20 & 3.8
\end{tabular}

The main parameters in Table 1 were obtained for the optimum working points for each amplifier stage with the following operating drain currents: $I_{\mathrm{d} 1}=I_{\mathrm{d} 2}=3.3 \mathrm{~mA}, I_{\mathrm{d} 3}=5.8 \mathrm{~mA}, I_{\mathrm{d} 4}=$ $6 \mathrm{~mA}$. Increasing the drain working currents to $10 \mathrm{~mA}$ does not lead to an increase of gain but significantly increases the noise temperature to $15 \mathrm{~K}$ with a total power consumption of $73 \mathrm{~mW}$. It is important to note that during the cLNA and qubit measurement experiments the dilution refrigerator was working stable with a constant $\mathrm{He}_{3} / \mathrm{He}_{4}$ mixture flow rate and with stable temperature values at each cooling stage.

\section{X-mon qubit characterization}

The implemented cryogenic LNA is dedicated for measurements in a frequency range from 6 to $12 \mathrm{GHz}$ and it was installed at the $4 \mathrm{~K}$ stage of the dilution refrigerator. The sensitivity and measurement speed of the full measurement setup is obviously defined by the signal-to-noise ratio and, thus, by the gain and the noise temperature of the cLNA.

The sample under study contained superconducting X-mon qubits coupled to coplanar quarter wavelength resonators. Each resonator was coupled to a single qubit. The sample was made at the BMSTU Nanofabrication Facility (FMN Laboratory, FMNS REC, ID 74300) using Al technology [29,30]. The $\mathrm{X}$-mon qubit was made of two $\mathrm{Al} / \mathrm{Al}_{x} \mathrm{O}_{y} / \mathrm{Al}$ parallel Josephson junctions forming a SQUID-type structure coupled to the main ground plane. The standard spectroscopy experiments were carried out using low input signal powers.

The qubit measurement setup is presented in Figure 5 where the designed and implemented amplifier is marked as cLNA. In order to decouple the sample from external noise sources we used two cryogenic circulators (marked as CC1 and CC2 in Figure 3) installed in series at the output of the sample and wideband cryogenic attenuators installed in a microwave input line, see Figure 5. The two circulators placed in series at the $10 \mathrm{mK}$ stage had a direct loss rate of about $4 \mathrm{~dB}$ and a return loss rate of about $40 \mathrm{~dB}$ in our measurement frequency range. Their main function was to isolate the sample from cLNA noise.

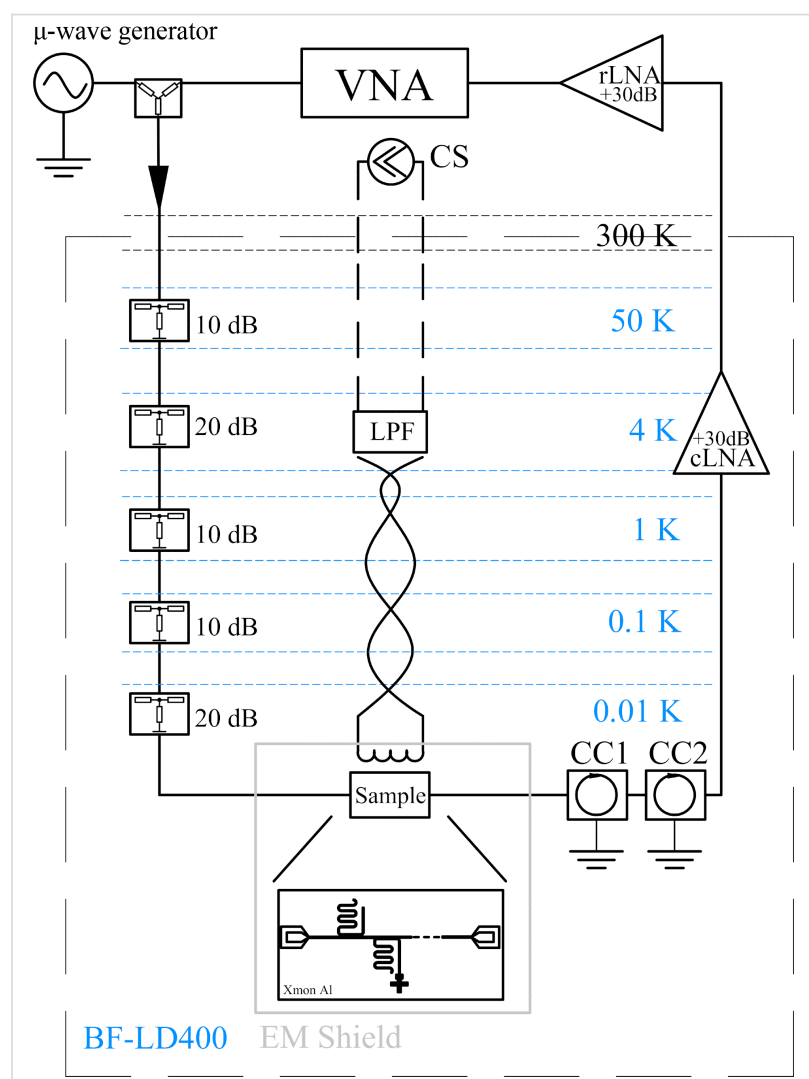

Figure 5: The X-mon qubit measurement setup. The implemented amplifier is marked as CLNA and the room-temperature amplifier is marked as rLNA. CC1 and CC2 are cryogenic circulators with $50 \Omega$ termination (LPF: low-pass filter for the superconducting coil line, CS: high-precision current source). The following setup was installed in a BlueFors BF-LD400 dilution refrigerator.

Wideband cryogenic attenuators (marked as $10 \mathrm{~dB}$ and $20 \mathrm{~dB}$ ) for room-temperature noise suppression and noise suppression 
of each next following temperature stage were placed according to the scheme in Figure 5. The VNA and a wideband stable microwave sweeping signal generator (marked as " $\mu$-wave" generator in Figure 5) were used for spectroscopy measurements. The standard heterodyne measurement method was used. A superconducting $\mathrm{Nb}$ coil was used for tuning the qubit eigenfrequency for each experiment. The coil was biased by means of a highly stable current source (marked as CS in Figure 5). The sample was placed in the copper sample holder with $\mathrm{Pb}$ and Mu-metal electromagnetic shields at the $10 \mathrm{mK}$ temperature stage. A two-stage differential low-pass filter (marked as LPF) with $10 \mathrm{kHz}$ bandwidth and wide stopband frequency range was implemented characterized and installed into the measurement system at the $4 \mathrm{~K}$ temperature stage of the diluton refrigerator. Since the power level of the measured signal applied to the sample was approximately $-120 \mathrm{dBm}$ and the gain of the cryogenic LNA was $30 \mathrm{~dB}$, an additional room-temperature lownoise microwave amplifier (marked as rLNA in Figure 5) was used. It was dedicated to increasing the signal level at the output of cLNA before data acquisition. The rLNA did not influence the signal-to-noise ratio of the measurement system since this parameter is mainly defined by the cLNA output noise. The rLNA noise figure corresponded to $2.1 \mathrm{~dB}$ with a gain value of about $40 \mathrm{~dB}$ for the frequency range from 6 to $13 \mathrm{GHz}$.

The transmission $\left|S_{21}\right|$ of the sample was measured and the resonance frequencies of resonators were defined in the frequency spectrum from 6 to $9 \mathrm{GHz}$. We defined a resonator fundamental frequency of $f_{0}=7.0554 \mathrm{GHz}$ and experimentally characterized the qubit responce. First, one-tone spectroscopy was carried out. The transmission of a sweeping microwave signal through the sample in a frequency range of 7.05-7.06 GHz for different DC bias currents, producing an external magnetic field, was measured. The obtained curve is shown in Figure 6.

Here, the horizontal axis is a sweeping DC magnetic field in current values, the vertical axis is the sweeping frequency of a probing microwave signal and the color intensity graph shows the normalized transmission amplitude $\left|S_{21}\right|$ in units of $\mathrm{dB}$, where 0 corresponds to a normalized maximum transmission. Due to the magnetic flux quantization the qubit characteristic has periodic behavior. The maximum shift of the resonance frequency corresponds to a so called "sweet spot" and the distance between two sweet spots corresponds to the magnetic flux quantum $\Phi_{0}$. We would like to mention that the current graph was obtained with $100 \mathrm{~Hz}$ of the intermediate frequency VNA filter bandwidth and for our experimental setup only four averages were required. In this sense, the cLNA reduces the measurement time of the experiment, which is especially important for preliminary qubit experiments.

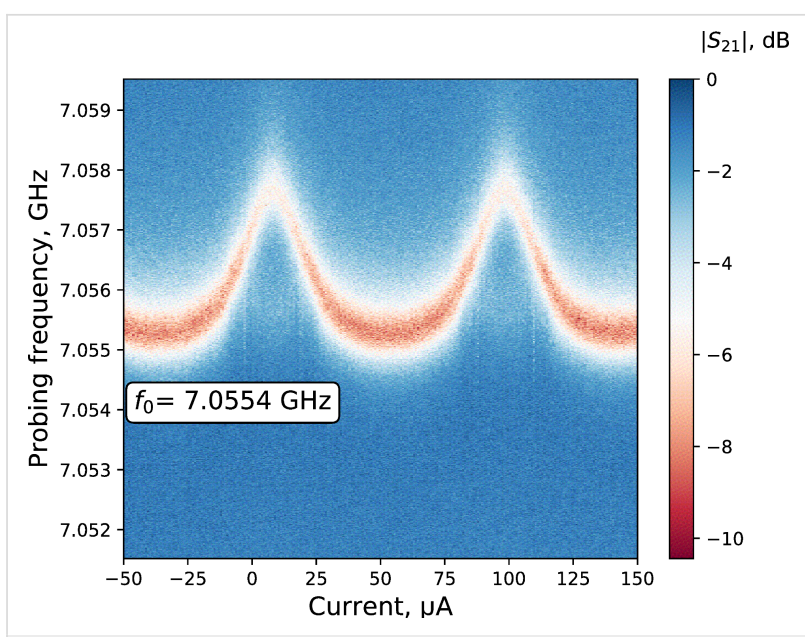

Figure 6: The qubit "sweet spot". The transmission (color intensity graph with the blue color corresponding to the maximum and the red color corresponding to the minimum) at the resonator resonance frequency depending on the external flux bias for the frequency range from 7.0515 to $7.0595 \mathrm{GHz}$ (vertical axis) and a constant probing power of $-120 \mathrm{dBm}$.

We have performed also a standard two-tone spectroscopy measurement using an additional stable wideband microwave generator, marked as " $\mu$-wave generator" in in Figure 5 and a resistive power combiner. We applied two microwave tones, that is, first a probing signal tone and then sweeping driving signal tones in a frequency range of 6.4-7.1 GHz. The powers applied to the sample after the input line attenuation were $-120 \mathrm{dBm}$ for the probing signal tone and $-80 \mathrm{dBm}$ for the driving signal tone, respectively. We defined the qubit spectrum depending on the external flux bias and found a minimum qubit energy gap of $\Delta$ $=6.625 \mathrm{GHz}$. The qubit spectrum as a function of the current is shown in Figure 7. Here, the transition $|g\rangle_{-}|e\rangle$ is shown.

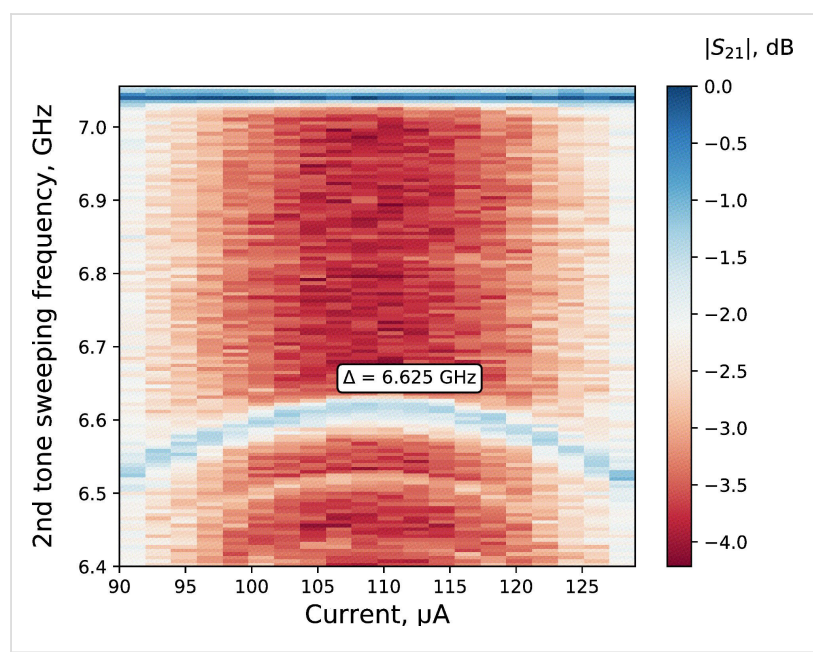

Figure 7: The qubit spectroscopy. The transmission dependence of the probing signal (color intensity graph) on the bias current (horizontal axes) for different values of driving signal frequency in a range from 6.4 to $7.1 \mathrm{GHz}$ (vertical axes). 


\section{Conclusion}

The characteristics of a low-noise cryogenic microwave amplifier and the measurement of a superconducting X-mon qubit are shown. The amplifier has a gain of more than $30 \mathrm{~dB}$ and a noise temperature lower than $6 \mathrm{~K}$ and is suitable for any high-sensitivity experiments in the presented frequency range. It is also necessary to mention that, during the qubit measurements with the implemented cLNA, the cryostat operating parameters, that is, the temperature of the stages and the cryostat $\mathrm{He}_{3} / \mathrm{He}_{4}$ mixture flow rate, were stable. The two-tone qubit spectroscopy experiment shown in this paper required about $48 \mathrm{~h}$ of continued measurements with the operating amplifier. The spectrum does not have any artifacts and shows a defined dependence on the external flux. Therefore, we conclude that the main parameters of the amplifier were stable during this time. From the noise curve it is seen that it is possible to achieve a noise temperature of $4 \mathrm{~K}$. This fact can be explained by a better noise matching for this particular cryogenic temperature.

\section{Acknowledgements}

The authors thank Ya. S. Greenberg and A. N. Sultanov for valuable discussions.

\section{Funding}

The research leading to these results has received funding from the Ministry of Science and Higher Education of the Russian Federation through the project number FSUN-2020-0007.

\section{ORCID ${ }^{\circledR}$ iDs}

Boris I. Ivanov - https://orcid.org/0000-0003-3410-349X llya L. Novikov - https://orcid.org/0000-0001-6328-9911 llya A. Rodionov - https://orcid.org/0000-0002-8931-5142 Aleksey G. Vostretsov - https://orcid.org/0000-0002-4753-4304

\section{References}

1. Hagmann, C.; Kinion, D.; Stoeffl, W.; van Bibber, K.; Daw, E.; Peng, H.; Rosenberg, L. J.; LaVeigne, J.; Sikivie, P.; Sullivan, N. S.; Tanner, D. B.; Nezrick, F.; Turner, M. S.; Moltz, D. M.; Powell, J.; Golubev, N. A. Phys. Rev. Lett. 1998, 80, 2043-2046. doi:10.1103/physrevlett.80.2043

2. Hoskins, J.; Crisosto, N.; Gleason, J.; Sikivie, P.; Stern, I.; Sullivan, N. S.; Tanner, D. B.; Boutan, C.; Hotz, M.; Khatiwada, R.; Lyapustin, D.; Malagon, A.; Ottens, R.; Rosenberg, L. J.; Rybka, G.; Sloan, J.; Wagner, A.; Will, D.; Carosi, G.; Carter, D.; Duffy, L. D.; Bradley, R.; Clarke, J.; O'Kelley, S.; van Bibber, K.; Daw, E. J. Phys. Rev. D 2016, 94, 082001. doi:10.1103/physrevd.94.082001

3. Brubaker, B. M.; Zhong, L.; Gurevich, Y. V.; Cahn, S. B.; Lamoreaux, S. K.; Simanovskaia, M.; Root, J. R.; Lewis, S. M.; Al Kenany, S.; Backes, K. M.; Urdinaran, I.; Rapidis, N. M.; Shokair, T. M.; van Bibber, K. A.; Palken, D. A.; Malnou, M.; Kindel, W. F.; Anil, M. A.; Lehnert, K. W.; Carosi, G. Phys. Rev. Lett. 2017, 118, 061302. doi:10.1103/physrevlett.118.061302
4. Wendin, G. Rep. Prog. Phys. 2017, 80, 106001 doi:10.1088/1361-6633/aa7e1a

5. Clarke, J.; Wilhelm, F. K. Nature 2008, 453, 1031-1042. doi:10.1038/nature07128

6. Martin, E. W.; Cundiff, S. T. Phys. Rev. B 2018, 97, 081301. doi:10.1103/physrevb.97.081301

7. Oelsner, G.; Andersen, C. K.; Rehák, M.; Schmelz, M.; Anders, S.; Grajcar, M.; Hübner, U.; Mølmer, K.; Il'ichev, E. Phys. Rev. Appl. 2017, 7, 014012. doi:10.1103/physrevapplied.7.014012

8. Macha, P.; van der Ploeg, S. H. W.; Oelsner, G.; Il'ichev, E.; Meyer, H.-G.; Wünsch, S.; Siegel, M. Appl. Phys. Lett. 2010, 96, 062503. doi:10.1063/1.3309754

9. Neilinger, P.; Oelsner, G.; Grajcar, M.; Ivanov, B. I.; Novikov, I. L.; Il'ichev, E. V. Tech. Phys. Lett. 2015, 41, 314-316. doi:10.1134/s1063785015040136

10. Il'ichev, E.; Oukhanski, N.; Wagner, T.; Meyer, H.-G.; Smirnov, A. Y.; Grajcar, M.; Izmalkov, A.; Born, D.; Krech, W.; Zagoskin, A. Low Temp. Phys. 2004, 30, 620-628. doi:10.1063/1.1789933

11. Wadefalk, N.; Mellberg, A.; Angelov, I.; Barsky, M. E.; Bui, S.; Choumas, E.; Grundbacher, R. W.; Kollberg, E. L.; Lai, R.; Rorsman, N.; Starski, P.; Stenarson, J.; Streit, D. C.; Zirath, H. IEEE Trans. Microwave Theory Tech. 2003, 51, 1705-1711. doi:10.1109/tmtt.2003.812570

12. Weinreb, S.; Bardin, J.; Mani, H.; Jones, G. Rev. Sci. Instrum. 2009, 80, 044702. doi:10.1063/1.3103939

13. Aja, B.; Schuster, K.; Schafer, F.; Gallego, J. D.; Chartier, S.; Seelmann-Eggebert, M.; Kallfass, I.; Leuther, A.; Massler, H.; Schlechtweg, M.; Diez, C.; Lopez-Fernandez, I.; Lenz, S.; Turk, S. IEEE Microwave Wireless Compon. Lett. 2011, 21, 613-615. doi:10.1109/Imwc.2011.2167502

14. Montazeri, S.; Grimes, P. K.; Tong, C.-Y. E.; Bardin, J. C. IEEE Trans. Appl. Supercond. 2017, 27, 1500605. doi:10.1109/tasc.2016.2631441

15. Ivanov, B. I.; Trgala, M.; Grajcar, M.; Il'ichev, E.; Meyer, H.-G. Rev. Sci. Instrum. 2011, 82, 104705. doi:10.1063/1.3655448

16. Ivanov, B. I.; Grajcar, M.; Novikov, I. L.; Vostretsov, A. G.; Il'ichev, E. Tech. Phys. Lett. 2016, 42, 380-383. doi:10.1134/s1063785016040076

17. Risacher, C.; Vassilev, V.; Pavolotsky, A.; Belitsky, V. IEEE Microwave Wireless Compon. Lett. 2003, 13, 262-264. doi:10.1109/Imwc.2003.815182

18. Bardin, J. C.; Weinreb, S. IEEE Microwave Wireless Compon. Lett. 2009, 19, 407-409. doi:10.1109/Imwc.2009.2020041

19. Cha, E.; Wadefalk, N.; Nilsson, P.-A.; Schleeh, J.; Moschetti, G.; Pourkabirian, A.; Tuzi, S.; Grahn, J. IEEE Trans. Microwave Theory Tech. 2018, 66, 4860-4869. doi:10.1109/tmtt.2018.2872566

20. Korolev, A. M.; Shnyrkov, V. I.; Shulga, V. M. Rev. Sci. Instrum. 2011, 82, 016101. doi:10.1063/1.3518974

21. Neilinger, P.; Trgala, M.; Grajcar, M. Cryogenics 2012, 52, 362-365. doi:10.1016/j.cryogenics.2012.02.006

22. Arakawa, T.; Nishihara, Y.; Maeda, M.; Norimoto, S.; Kobayashi, K. Appl. Phys. Lett. 2013, 103, 172104. doi:10.1063/1.4826681

23. Tracy, L. A.; Luhman, D. R.; Carr, S. M.; Bishop, N. C.; Ten Eyck, G. A.; Pluym, T.; Wendt, J. R.; Lilly, M. P.; Carroll, M. S. Appl. Phys. Lett. 2016, 108, 063101. doi:10.1063/1.4941421

24. Oukhanski, N.; Grajcar, M.; Il'ichev, E.; Meyer, H.-G. Rev. Sci. Instrum. 2003, 74, 1145. doi:10.1063/1.1532539

25. Ivanov, B. I.; Novikov, I. L.; Sultanov, A. N.; Greenberg, Y. S.; Krivetskii, A. V.; Vostretsov, A. G.; Il'ichev, E. JETP Lett. 2016, 103, 425-430. doi:10.1134/s0021364016060047 
26. Novikov, I. L.; Ivanov, B. I.; Sultanov, A. N.; Greenberg, Y. S.; Il'ichev, E. V. Phys. Solid State 2016, 58, 2155-2159.

doi:10.1134/s1063783416110287

27. Oelsner, G.; van der Ploeg, S. H. W.; Macha, P.; Hübner, U.; Born, D.; Anders, S.; Il'ichev, E.; Meyer, H.-G.; Grajcar, M.; Wünsch, S.;

Siegel, M.; Omelyanchouk, A. N.; Astafiev, O. Phys. Rev. B 2010, 81, 172505. doi:10.1103/physrevb.81.172505

28. Y-Factor Method for Noise Figure Measurement Accuracy; Keysight Application Note 57-2.

https://www.keysight.com/ru/ru/assets/7018-06829/application-notes/59 52-3706.pdf (accessed Aug 28, 2020).

29. Moskalenko, I. N.; Besedin, I. S.; Tsitsilin, I. A.; Mazhorin, G. S.; Abramov, N. N.; Grigor'ev, A.; Rodionov, I. A.; Dobronosova, A. A.; Moskalev, D. O.; Pishchimova, A. A.; Ustinov, A. V. JETP Lett. 2019, 110, 574-579. doi:10.1134/s0021364019200074

30. Rodionov, I. A.; Baburin, A. S.; Gabidullin, A. R.; Maklakov, S. S.; Peters, S.; Ryzhikov, I. A.; Andriyash, A. V. Sci. Rep. 2019, 9, 12232. doi:10.1038/s41598-019-48508-3

\section{License and Terms}

This is an Open Access article under the terms of the Creative Commons Attribution License (https://creativecommons.org/licenses/by/4.0). Please note that the reuse, redistribution and reproduction in particular requires that the authors and source are credited.

The license is subject to the Beilstein Journal of Nanotechnology terms and conditions: (https://www.beilstein-journals.org/bjnano)

The definitive version of this article is the electronic one which can be found at: https://doi.org/10.3762/bjnano.11.131 